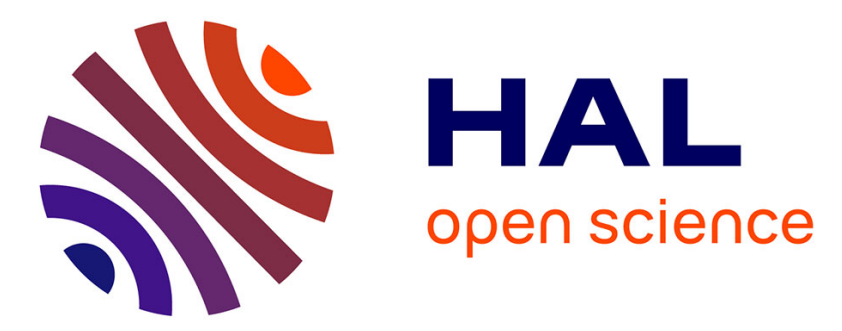

\title{
Investigation of 3D surface acoustic waves in granular media with 3-color digital holography
}

Mathieu Leclerc, Pascal Picart, Guillaume Penelet, Vincent Tournat

\section{To cite this version:}

Mathieu Leclerc, Pascal Picart, Guillaume Penelet, Vincent Tournat. Investigation of 3D surface acoustic waves in granular media with 3-color digital holography. Journal of Applied Physics, 2017, 121 (4), pp.045112. 10.1063/1.4974950 . hal-02057081

\section{HAL Id: hal-02057081 \\ https://hal-univ-lemans.archives-ouvertes.fr/hal-02057081}

Submitted on 5 Mar 2019

HAL is a multi-disciplinary open access archive for the deposit and dissemination of scientific research documents, whether they are published or not. The documents may come from teaching and research institutions in France or abroad, or from public or private research centers.
L'archive ouverte pluridisciplinaire HAL, est destinée au dépôt et à la diffusion de documents scientifiques de niveau recherche, publiés ou non, émanant des établissements d'enseignement et de recherche français ou étrangers, des laboratoires publics ou privés. 
Investigation of 3D surface acoustic waves in granular media with 3-color digital holography

Mathieu Leclercq, Pascal Picart, Guillaume Penelet, and Vincent Tournat

Citation: J. Appl. Phys. 121, 045112 (2017); doi: 10.1063/1.4974950

View online: http://dx.doi.org/10.1063/1.4974950

View Table of Contents: http://aip.scitation.org/toc/jap/121/4

Published by the American Institute of Physics

\section{AIP Journal of Applied Physics}

INTRODUCING INVITED PERSPECTIVES Ultrafast magnetism and $\mathrm{THz}$ spintronics Authors: Jakob Walowski and Markus Münzenberg 


\title{
Investigation of 3D surface acoustic waves in granular media with 3-color digital holography
}

\author{
Mathieu Leclercq, ${ }^{1}$ Pascal Picart, ${ }^{1,2, a)}$ Guillaume Penelet, ${ }^{1}$ and Vincent Tournat ${ }^{1, b)}$ \\ ${ }^{1}$ Université du Maine, CNRS UMR 6613, LAUM, Av. O. Messiaen, 72805 Le Mans, France \\ ${ }^{2}$ Ecole Nationale Supérieure d'Ingénieurs du Mans, Rue Aristote, 72805 Le Mans, France
}

(Received 28 October 2016; accepted 13 January 2017; published online 31 January 2017)

\begin{abstract}
This paper reports the implementation of digital color holography to investigate elastic waves propagating along a layer of a granular medium. The holographic set-up provides simultaneous recording and measurement of the $3 \mathrm{D}$ dynamic displacement at the surface. Full-field measurements of the acoustic amplitude and phase at different excitation frequencies are obtained. It is shown that the experimental data can be used to obtain the dispersion curve of the modes propagating in this granular medium layer. The experimental dispersion curve and that obtained from a finite element modeling of the problem are found to be in good agreement. In addition, full-field images of the interaction of an acoustic wave guided in the granular layer with a buried object are also shown. Published by AIP Publishing. [http://dx.doi.org/10.1063/1.4974950]
\end{abstract}

\section{INTRODUCTION}

In the past decade, digital holographic imaging has been developed at an accelerating pace and impacted a wide range of domains, such as microscopy ${ }^{1-11}$ fluid mechanics, ${ }^{12,13}$ or photomechanics. ${ }^{14,15,17}$ Phase imaging measures the optical path length map associated with transparent specimens (transmission illumination) or opaque surfaces (reflection illumination) and translates these data into relevant information. Information can be of various nature, such as biomedical, ${ }^{3,4,7,8}$ topology at nanoscale, ${ }^{5,11}$ flows and vortices, ${ }^{13,14}$ material properties, ${ }^{15,16}$ surface shape, ${ }^{17}$ polarization, ${ }^{18}$ displacement field, ${ }^{19,20}$ or vibrations. ${ }^{21-24}$ Recently, a real-time three-dimensional deformation measurement based on threecolor digital Fresnel holography and simultaneous recording with a color sensor was demonstrated. ${ }^{20}$ Three-color holograms may thus be simultaneously recorded by using a simple experimental scheme without any need for sequential recording or the spatial multiplexing of holograms. Such a method provides an opportunity to investigate, with a fullfield capability, the acoustic waves propagating along the surface of a granular medium. Especially, the three dimensional probing may give information on the $3 \mathrm{D}$ character of the dynamic movements.

In previous works, we reported the use of two-color digital holography as a first approach to investigate soft modes in a three dimensional granular medium. ${ }^{25}$ Soft modes or floppy modes in a granular medium are collective modes of vibration with vanishing frequency, involving several grain displacement and rotation at low energy elastic cost. ${ }^{26}$ However, soft modes were not observable without any doubt since an approximation on the out-of-plane movement was used to determine the 3D components of the acoustic waves. In this paper, a three-color holographic probe giving a

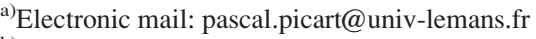

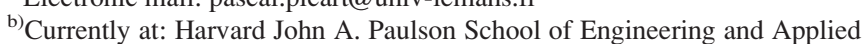
Science, Harvard University, Cambridge, Massachusetts 02138, USA.
}

simultaneous evaluation of the acoustic waves propagating along the surface of a granular layer is presented. Contrary to the use of a point wise laser vibrometer that detects outof-plane movements at the surface of the medium, ${ }^{27,30}$ the holographic probes provide a full-field measurement, without any scanning or sequential measurement. In addition, the use of several illumination beams leads to the measurement of the three components of the dynamic displacement. ${ }^{20}$ Such set-up is thus quite adapted to collect spatial information and measure the dispersion properties of the acoustic modes.

We present the implementation of the three color digital holographic method for the characterization of the guided waves inside a granular layer containing a property gradient. The 3D holographic probe is applied to study a granular medium in the form of a layer of $35 \mathrm{~mm}$ depth. The interest for the study of granular layers is related to the possible analogy, at the scale of a laboratory, with ground layers and the geophysics problems associated. In addition, the complexity of such a waveguide, which includes an elasticity gradient along its depth, ${ }^{31,32,34}$ is of interest for wave physics.

This paper is organized as follows: Section II presents the 3D digital holographic probe and discusses on the measurement of the three components of the displacement at the surface of the granular medium. Section III presents the experimental set-up, which is based on the use of three laser lines, and Section IV deals with the experimental procedure. In Section V, we give few experimental results showing fullfield images of the amplitude and phase of propagating acoustic waves; the dispersion curve is then extracted from the holographic data. In Section VI, a comparison between experimental results and a finite element modeling of the problem is provided; the comparison of the dispersion curves shows a good agreement. Section VII presents results obtained at excitation frequencies for which elastic waves with 3-component displacements are existing. Section VIII shows full-field images of the interaction of an acoustic 
wave with a buried object. Finally, Section IX draws conclusion and perspectives of this study.

\section{PRINCIPLE OF THE 3D HOLOGRAPHIC PROBE}

The classic holographic scheme is based on the interference between a reference wave, which can be plane or spherical, and an object wave, which is scattered and then propagated from the surface to the sensor plane. The interference is recorded as a digital hologram, $H$, at the recording plane by a matrix sensor. Note that $R(x, y)$ $=a_{R}(x, y) \exp \left(-2 i \pi\left(u_{0} x+v_{0} y\right)\right)$ refers to the reference wave, with $\left(u_{0}, v_{0}\right)$ being its spatial frequencies, at the recording plane. Note also that $O\left(x, y, d_{0}\right)=\{A * h\}(x, y)$ is the object wave at the same plane located at a distance $d_{0}$ from the object (* means convolution). We have $A(x, y)=A_{0}(x, y) \exp \left(i \Psi_{0}(x, y)\right)$ for the object wave at the object plane, and $h\left(x, y, d_{0}\right)$ is the impulse response of free space propagation along the distance $d_{0}{ }^{35}$ The recorded hologram can be written as ${ }^{1,36}$

$$
H=|R|^{2}+|O|^{2}+R^{*} O+R O^{*} .
$$

The hologram is then numerically reconstructed, at a distance $d_{r}$ that can be different from $d_{0}{ }^{36,37}$ Using a digital sensor, the reconstructed hologram is the discrete version of ${ }^{38}$

$$
A_{r}=H * h(x, y) .
$$

There are several possibilities for the reconstruction, depending on the interpretation of Equation (2). The first one is the convolution approach, which considers 2 as an exact convolution formula. ${ }^{38,40}$ The second one is the Fresnel approach, where 2 is considered as the 2D Fresnel discrete tranform. ${ }^{36,38}$

The result of the different reconstructions is a complex amplitude, with a numerical sampling related to the number of pixels used in the reconstruction algorithm. From this complex amplitude, the amplitude and the phase of the optical wave can be obtained. The complex amplitude obtained using the Fresnel approach is given by ${ }^{36-38}$

$$
\begin{aligned}
A_{r}\left(x, y, d_{r}\right)= & -\frac{i \exp \left(2 i \pi d_{r} / \lambda\right)}{\lambda d_{r}} \exp \left(\frac{i \pi}{\lambda d_{r}}\left(x^{2}+y^{2}\right)\right) \\
& \times \sum_{-M / 2-N / 2}^{M / 2} \sum^{N / 2} H\left(n p_{x}, m p_{y}\right) \exp \left(\frac{i \pi}{\lambda d_{r}}\left(n^{2} p_{x}^{2}+m^{2} p_{y}^{2}\right)\right) \\
& \times \exp \left(-\frac{2 i \pi}{\lambda d_{r}}\left(n p_{x} x+m p_{y} y\right)\right) .
\end{aligned}
$$

The amplitude and phase are calculated with

$$
\begin{aligned}
& A_{r}(x, y)=\left|A_{r}(x, y)\right| \\
& \psi_{r}(x, y)=\arg \left(A_{r}(x, y)\right)=\tan ^{-1}\left(\frac{\Im\left[A_{r}(x, y)\right]}{\Re\left[A_{r}(x, y)\right]}\right) \bmod 2 \pi .
\end{aligned}
$$

The phase image is the key for contact-less measurements. When the object is deformed or moves, the light path from the object to the sensor varies. The phase variation, $\Delta \varphi$, induced by the optical path variation is related to the $3 \mathrm{D}$ displacement vector $\mathbf{U}=\left(\mathbf{U}_{x}, \mathbf{U}_{y}, \mathbf{U}_{z}\right)$ according to ${ }^{14,39}$

$$
\Delta \varphi=\frac{2 \pi}{\lambda} \mathbf{U} \cdot\left(\mathbf{K}_{e}-\mathbf{K}_{o}\right)=\frac{2 \pi}{\lambda} \mathbf{U} \cdot \mathbf{S},
$$

where $\mathbf{K}_{e}$ is the "illumination" vector, giving the direction of the object's illumination, $\mathbf{K}_{o}$ is the "observation" vector, giving the direction towards the recording plane, and $\lambda$ is the illumination wavelength. The sensitivity vector $\mathbf{S}$, represented by $\mathbf{S}=\mathbf{K}_{e}-\mathbf{K}_{o}$, indicates the displacement direction where the optical path variation is maximum.

The 3D probe properties are based upon the relation between the 3D displacement vector of the object, $\mathbf{U}$, the phase differences $\Delta \varphi$ for different object states, and the sensitivity vector $\mathbf{S}$. Figure 1 illustrates these geometrical vectors in the classical holographic scheme.

Considering the notations in Figure 1, the vectors are (angles are oriented)

$$
\begin{aligned}
\mathbf{U} & =u_{x} \mathbf{i}+u_{y} \mathbf{j}+u_{z} \mathbf{k} \\
\mathbf{K}_{\mathbf{o}} & =\mathbf{k} \\
\mathbf{K}_{\mathbf{e}}^{\lambda} & =-\cos \left(\theta_{y z}^{\lambda}\right) \sin \left(\theta_{x z}^{\lambda}\right) \mathbf{i}-\sin \left(\theta_{y z}^{\lambda}\right) \mathbf{j}-\cos \left(\theta_{y z}^{\lambda}\right) \cos \left(\theta_{x z}^{\lambda}\right) \mathbf{k} .
\end{aligned}
$$

The use of three different wavelengths, $\lambda_{R}, \lambda_{G}, \lambda_{B}$, leads to three equations, which allows measuring the three components $u_{x}, u_{y}, u_{z}$. Equation (5) leads to a matrix relation. Noting A the matrix from scalar product in 5, and inverting relation 5 leads to the displacement field in the three directions of space $x, y$, and $z$

$$
\left(\begin{array}{l}
u_{x} \\
u_{y} \\
u_{z}
\end{array}\right)=\frac{1}{2 \pi} \mathbf{A}^{-1}\left(\begin{array}{c}
\lambda_{R} \Delta \varphi_{\lambda_{R}} \\
\lambda_{G} \Delta \varphi_{\lambda_{G}} \\
\lambda_{B} \Delta \varphi_{\lambda_{B}}
\end{array}\right) .
$$

So, the use of three wavelengths provides the opportunity to measure and visualize 3D acoustic waves propagating at the surface of a granular medium.

\section{EXPERIMENTAL SETUP}

The 3-color digital holographic setup for the study of the elastic waves in granular layers is described in Fig. 2. Three continuous lasers, with, respectively, wavelengths at $\lambda_{R}=660, \lambda_{G}=532$, and $\lambda_{B}=457$ nanometers, are used to illuminate the object. The laser beams first pass through acousto-optic modulators, allowing the synchronization of the illumination and reference beams with the excitation signal (detailed in Section IV).
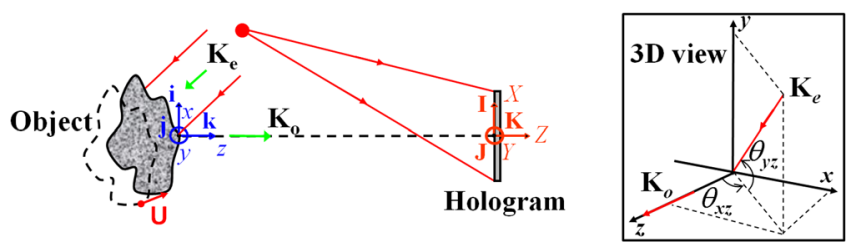

FIG. 1. Basic scheme for the 3D holographic probe. 


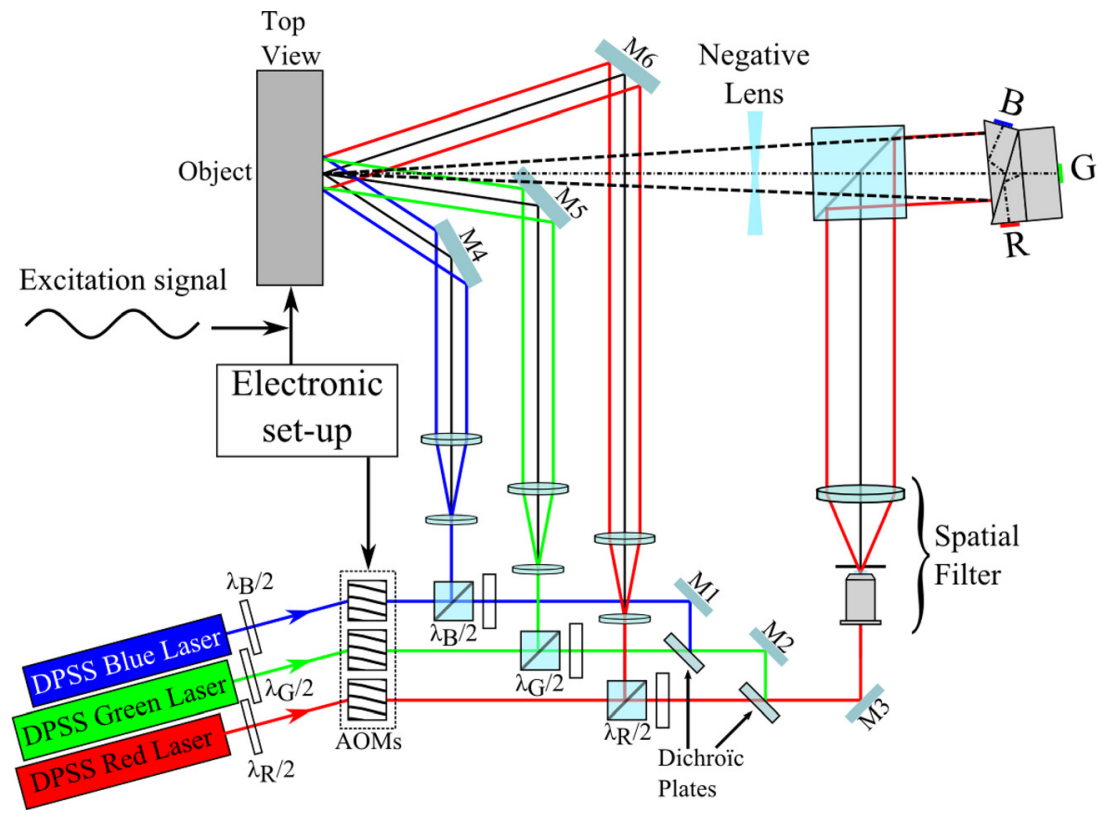

FIG. 2. Experimental setup using three laser wavelengths. AOMs: AcoustoOptic Modulators And M1-M6: Mirrors.

The three beams are then separated using polarizing cubes in order to give three illuminating beams and three reference beams, the power of which are adjusted by using half-wave plates. Another $\lambda / 2$ plate placed after the polarizing cube rotates the polarization by $90^{\circ}$ so that the object and reference beams are co-polarized. The reference beams are combined using dichroic plates, before being spatially filtered, and guided towards the sensor with the help of the $50 \%$ cube located in front of the sensor. The spatial filters are composed of a microscope objective, a $20 \mu \mathrm{m}$ diameter hole, and an achromatic lens, the focal plane of which is located at the microscopic hole. The sensor is a 3-CCD camera, which separates the three wavelengths using a dichroic prism towards three CCD sensors, each composed of 1024 $\times 1344$ pixels sized $6.45 \mu \mathrm{m}$. This leads to a simultaneous recording of the three color holograms and so provides a simultaneous 3D displacement measurement.

The object beams illuminate the useful area from three directions, making the matrix A from Equation (7) invertible. The six angles from Equation (6) are $\theta_{x z}^{R}=-0.679 \mathrm{rad}$ $=-38.89^{\circ}, \theta_{x z}^{G}=-2.022 \mathrm{rad}=-115.85^{\circ}, \theta_{x z}^{B}=1.035 \mathrm{rad}$ $=59.3^{\circ}, \theta_{y z}^{R}=0.304 \mathrm{rad}=17.42^{\circ}, \theta_{y z}^{G}=0.423 \mathrm{rad}=24.24^{\circ}$ and $\theta_{y z}^{B}=0.514 \mathrm{rad}=29.45^{\circ}$, almost forming a trihedron.
The object under interest is a layer, $35 \mathrm{~mm}$ deep, of a granular medium, composed of glass beads of $150 \mu \mathrm{m}$ in diameter and placed inside a container, which is $350 \mathrm{~mm}$ wide and $450 \mathrm{~mm}$ long. The illuminated area is sized $10 \times 10 \mathrm{~cm}^{2}$. A mirror is located beyond the container with a $45^{\circ}$ angle so as to reflect the diffracted light towards the 3CCD camera. Figure 3 presents the illumination geometry. The granular layer is located at a distance $d_{0}=-1200 \mathrm{~mm}$ from the sensor.

In order to reduce the spatial frequency spectrum of the object and to compact the setup, a negative lens with a $-250 \mathrm{~mm}$ focal length is placed in the path of the diffracted illumination beam, just before the $50 \%$ cube located in front of the sensor. ${ }^{41,42}$ So, a virtual object is created in front of the sensor, at a reduced distance compared to the distance imposed by the Shannon criteria. ${ }^{37}$

The use of a negative lens brings chromatic aberrations, ${ }^{43,44}$ which are suppressed using the technique presented in Reference 45 . The method is based on a modified zero-padding algorithm ${ }^{46,47}$ and a convolution algorithm. ${ }^{40}$ This correction leads to the superimposition of the three color reconstructed images, with no error in the position and no size difference. ${ }^{45}$

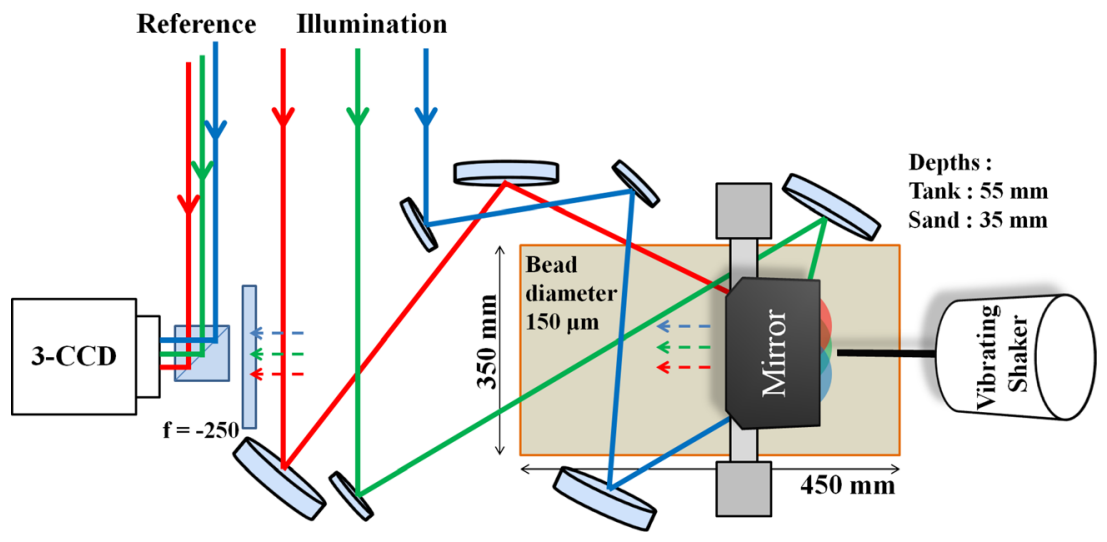

FIG. 3. Scheme of the illumination part above the granular layer. 
The reduced reconstruction distances for the three wavelengths (red, green, and blue) are, respectively, -340, -334, and $-328 \mathrm{~mm}$.

The medium is excited using a vibrating shaker attached to a metal stick buried in the granular layer. The acoustic excitation is provided to the shaker using a function generator, which also provides the synchronous TTL signal to the acousto-optic modulators. The acoustic excitation is sinusoidal with a frequency varying between $800 \mathrm{~Hz}$ and $2000 \mathrm{~Hz}$. The applied voltage is a few volts (typ. 1-4 V) and is injected to an amplifier linked to the shaker. The amplifier is set so that at the maximum adjustment, the voltage from the amplifier stays below the voltage at which the displacement amplitude becomes high enough to induce permanent modification of the medium. The voltage can then be adjusted on the generator without modifying the amplifier and ensuring a nondestructive regime of wave operation.

\section{MEASUREMENT PROCEDURE}

To determine the 3 dynamic components of the acoustic displacement, at least three phase differences must be recorded for each frequency. The object illumination must be synchronous with the excitation signal. This synchronization is performed by the acousto-optic modulators. The TTL signal is provided to them by the same generator, which provides the excitation signal. When the TTL signal is equal to 0 , the beam crossing the modulator is not deviated, and it does not pass through the separating cube placed after the modulator (see Figure 4). However, when the TTL signal is equal to 1 , the beam is deviated, allowing the object to be illuminated, and the hologram is recorded with a temporal shift controlled by the function generator.

The TTL (Transistor-Transistor Logic) signal driving the AOMs (Acousto Optic Modulator) has a frequency equal to that of the excitation (maximum period: $1 / 800 \mathrm{~ms}$ ), with a pulse width equal to $1 \%$ of the period. The recording by the sensor is performed over a $2 \mathrm{~s}$ integration time; the TTL signal reaches periodically $5 \mathrm{~V}$, the AOMs deviates the beams several times during this period, between 8000 and 2000 times, and the sensor records between 800 and 2000 times the phenomenon for one single hologram, and this is for each excitation frequency. Thus, the cyclic ratio is 0.01 , corresponding to the impulse regime. ${ }^{48}$ So, there is a "freezing"
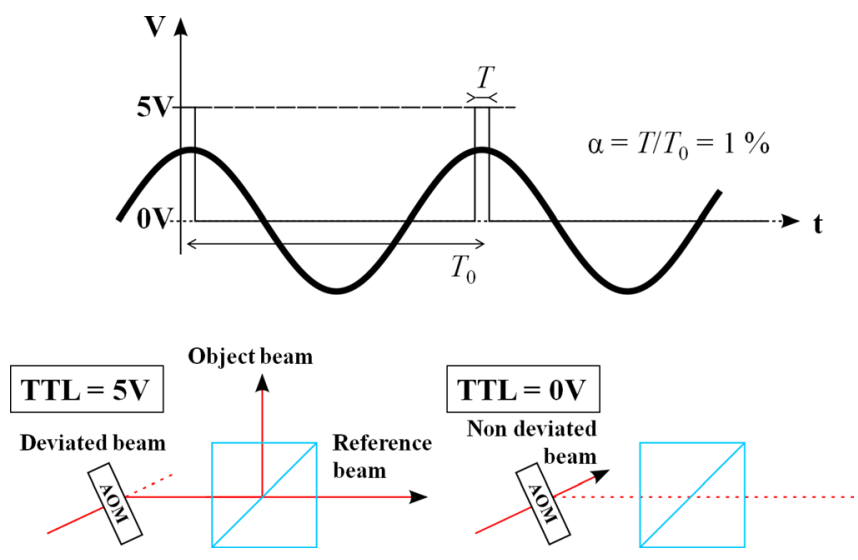

FIG. 4. Scheme of the implementation of acousto-optic modulators. of the object during the recording, even with such a dynamic phenomenon (stroboscopic recording). ${ }^{48}$

The procedure consists in recording three holograms, phase shifted by a quarter of the excitation period. The three components of the displacement field can then be written as a function of the synchronization phase

$$
u_{c}(k)=u_{c} \sin \left(\omega_{0} t_{0}+\varphi_{c}+(k-1) \frac{\pi}{2}\right),
$$

with $c=x, y, z$ the spatial directions, $u_{c}$ the amplitude of the acoustic displacement, $\varphi_{c}$ the phase of the acoustic displacement, $\omega_{0}$ the excitation pulsation, and $t_{0}$ the time origin of the recording. $k$ is an integer equal to 1,2 , or 3 , related to the number of the hologram phase-shifted versus the excitation. Using Equation (7), nine displacement differences are calculated (three for each spatial direction $x, y$, and $z$ )

$$
\begin{aligned}
\left(\begin{array}{l}
u_{x}(m)-u_{x}(n) \\
u_{y}(m)-u_{y}(n) \\
u_{z}(m)-u_{z}(n)
\end{array}\right) & =\left(\begin{array}{c}
\Delta u_{x}(m n) \\
\Delta u_{y}(m n) \\
\Delta u_{z}(m n)
\end{array}\right) \\
& =\frac{1}{2 \pi} \mathbf{A}^{-1}\left(\begin{array}{c}
\lambda_{R} \Delta \varphi_{\lambda_{R}}(m n) \\
\lambda_{G} \Delta \varphi_{\lambda_{G}}(m n) \\
\lambda_{B} \Delta \varphi_{\lambda_{B}}(m n)
\end{array}\right),
\end{aligned}
$$

where $\Delta \varphi_{\lambda}(m n)$ represents the optical phase difference and $\Delta u_{c}(m n)$ represents the displacement difference, between the $m$-th and the $n$-th recording $([m, n] \in\{1,2,3\})$.

The algorithm for measuring the acoustic displacement amplitude and phase as a function of the displacement differences is given by ${ }^{48}$

$$
\left\{\begin{array}{l}
u_{c}=\frac{1}{2} \sqrt{\Delta u_{c}^{2}(13)+\left[\Delta u_{c}(23)+\Delta u_{c}(21)\right]^{2}} \\
\varphi_{c}=\arctan \left[\frac{\Delta u_{c}(13)}{\Delta u_{c}(23)+\Delta u_{c}(21)}\right](\bmod 2 \pi) .
\end{array}\right.
$$

The experimental method can be summarized in 5 steps. First, record the color holograms for each phase shift $(0$, $\pi / 2, \pi)$; second, extract the three optical phases corrected from chromatic aberrations for the three acoustic phase shifts $\left(\varphi_{R}(k), \varphi_{G}(k), \varphi_{B}(k), k=(1,2,3)\right)$; third, calculate the three optical phase differences for each wavelength $\left(\Delta \varphi_{\lambda}(13), \Delta \varphi_{\lambda}(21), \Delta \varphi_{\lambda}(23)\right)$; fourth, estimate the nine displacement differences (Equation (9)); and finally, measure the acoustic displacement amplitude and phase for the 3 components (Equation (10)).

This experimental procedure was applied for excitation frequencies between $800 \mathrm{~Hz}$ and $2000 \mathrm{~Hz}$, with a $100 \mathrm{~Hz}$ step, for which the granular medium can be considered as an elastic wave guide. Section V presents the experimental results obtained using the $3 \mathrm{D}$ holographic probe.

\section{EXPERIMENTAL RESULTS}

Figures 5 and 6 show sets of modulo- $2 \pi$ optical phase differences obtained from color holograms of the granular medium surface at excitation frequencies between $800 \mathrm{~Hz}$ 


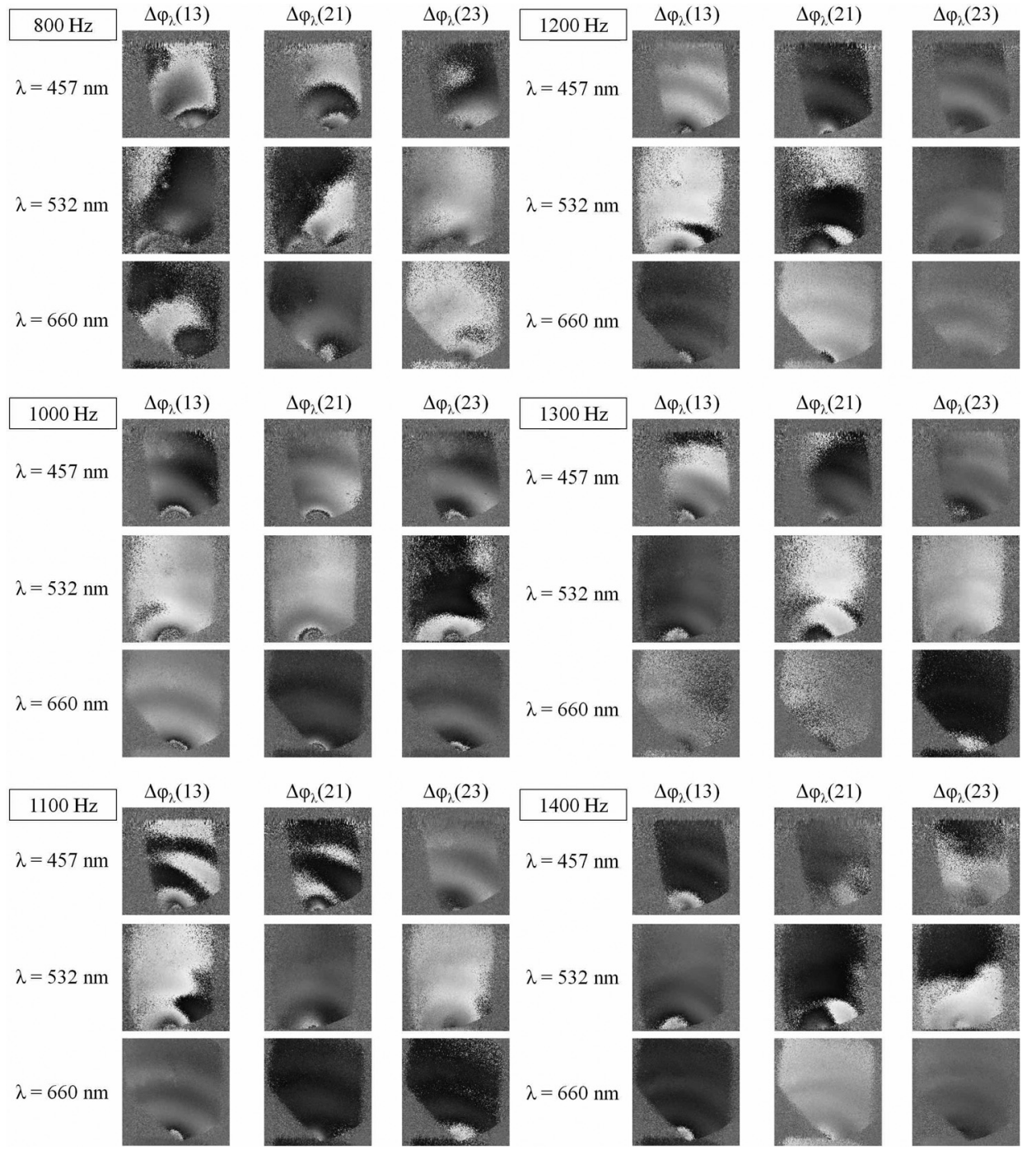

FIG. 5. Phase differences for the three wavelengths $(\lambda=457,532$, and $660 \mathrm{~nm})$ and for six excitation frequencies (from 800 to $1400 \mathrm{~Hz}$ ). and $2000 \mathrm{~Hz}$ for the three optical wavelengths and for the three acoustic phase shifts $(0, \pi / 2$, and $\pi)$.

On both figures, bright and dark fringes can be seen, which indicates the presence of waves inside the granular medium. These fringes depend on the excitation frequency of the metallic stick exciter buried inside the medium.

Figure 7 shows the results along the $z$ direction for the acoustic displacement at the surface, obtained for an excitation frequency of $1100 \mathrm{~Hz}$. Amplitudes between 0 and 50 nanometers are visible in the observation area, sized approximately $9 \times 9 \mathrm{~cm}^{2}$. The dark (blue in the colormap) lines on the left image are the lowest amplitude lines of the vibration, and there exists a strong correlation between these low amplitude lines and the phase jumps visible on the right image. This indicates that each line corresponds to a sign change for the acoustic amplitude, showing the existence of waves in the granular medium layer.

Figure 8 presents the results obtained for an excitation frequency of $1400 \mathrm{~Hz}$, for the $z$ component of the acoustic displacement. It is understood that the inter-fringe space is smaller than in Figure 7, expected results considering that the acoustic wavelength diminishes when the acoustic frequency increases.
Experimental and theoretical studies on the propagation of acoustic guided waves inside granular layers ${ }^{27-34}$ showed that the propagating modes along the surface, excited the same way as in our experiment, are modes guided due to the free surface and the elastic property gradient. Furthermore, these wave modes are polarized along the sagittal plane (such as Rayleigh waves) or can be shear-horizontal modes. The usual depth profile of these modes at $\sim 1 \mathrm{kHz}$ extends to more than $10 \mathrm{~cm}$ typically. In the present experiment, the granular layer depth is $3.5 \mathrm{~cm}$. The modes propagating along the surface are consequently not only guided by the elastic property gradient and the free surface but also by the bottom of the container, considered rigid here. Direct reflections from the bottom of the longitudinal waves excited by the source are, in principle, visible in the illuminated area of the surface, leading to possible acoustic interferences. Consequently, in the present configuration, the distinction between sagittally polarized guided modes and purely longitudinal reflected or guided waves is not straightforward, especially in the near field of the acoustic source.

If the relation between the wavelength and the frequency is established, one may measure the dispersion relation (between the pulsation and the wave number or equivalently 


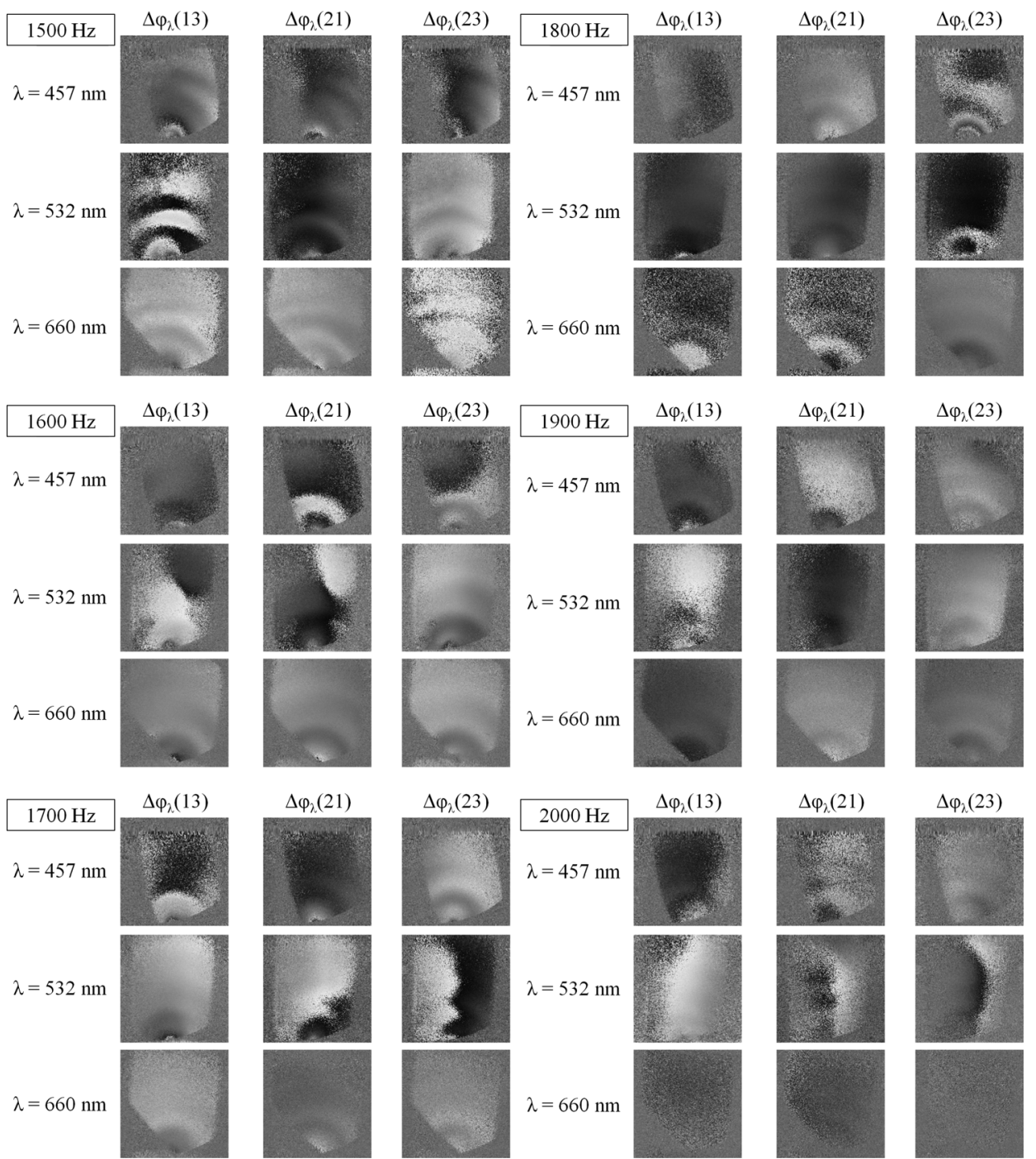

FIG. 6. Phase differences for the three wavelengths $(\lambda=457,532$, and $660 \mathrm{~nm}$ ) and for six excitation frequencies (from 1500 to $2000 \mathrm{~Hz}$ ).

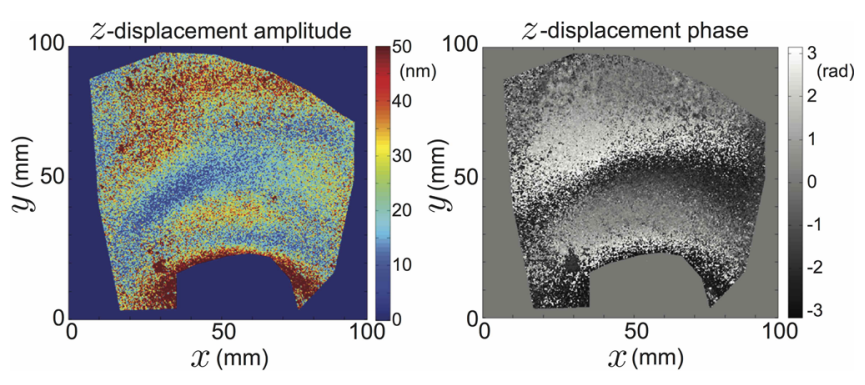

FIG. 7. Displacement at the surface along the $z$ direction. Amplitude (left) and phase (right) images for an excitation frequency of $1100 \mathrm{~Hz}$.

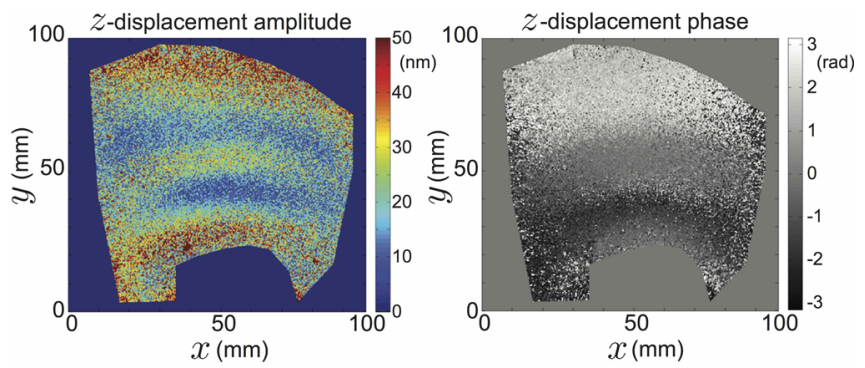

FIG. 8. Displacement at the surface along the $z$ direction. Amplitude (left) and phase (right) images for an excitation frequency of $1400 \mathrm{~Hz}$. between the frequency and the inverse of the wavelength) of the acoustic modes propagating along the surface of the granular layer. In order to quantify the observation of the inter-fringe distances (associated with the acoustic wavelength) for different acoustic frequencies, the amplitude profiles along the $z$ direction are plotted for each excitation frequency. Figure 9 shows an example of such profiles, for an excitation frequency of $1300 \mathrm{~Hz}$. Using such profiles, the apparent spatial wavelengths $\lambda_{a c}$ of the acoustic wave can be obtained. The propagative character of the observed mode (as opposed to a resonant mode with stationary waves) is confirmed by the observation of an acoustic displacement amplitude, which does not vary significantly when the excitation frequency is modified. Thus, the measured inter-fringe space corresponds to a fraction of the propagative mode wavelength.

Because of the measurement noise at these displacement amplitude scales, the inter-fringe distance is difficult to extract. In order to show the reader the influence of the image processing, the spatial wavelengths (inter-fringe distance) are extracted from experiments using three different methods. The first one is manual and graphical, with the inter-fringe distance being extracted from the manual pointing of the first and second deep blue color regions (corresponding to the 

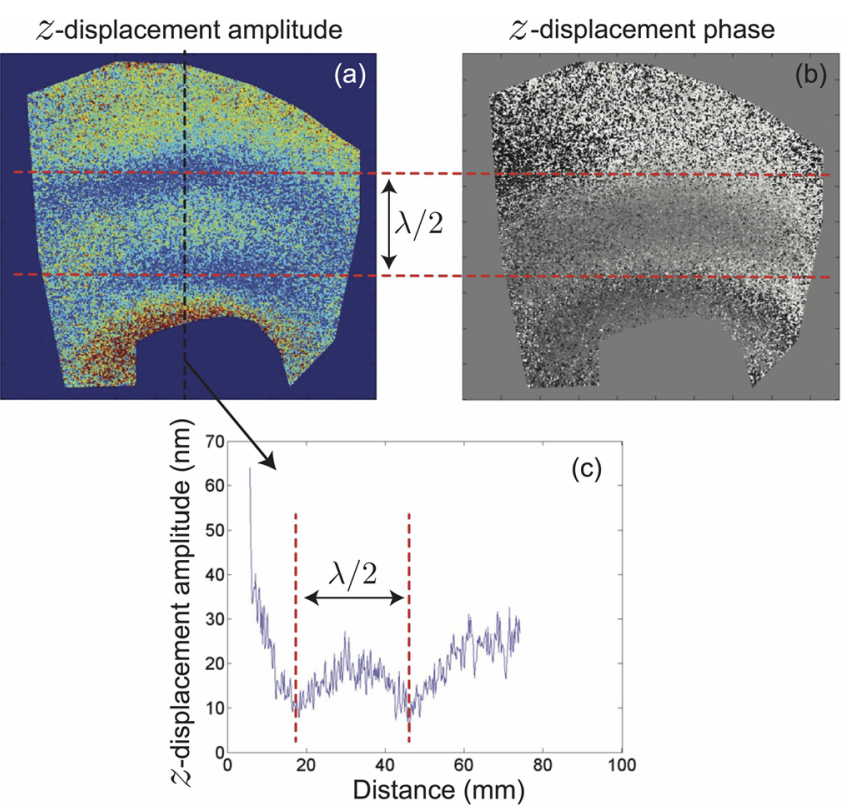

FIG. 9. $z$-displacement amplitude image (a), z-displacement phase image (b), and $z$-displacement amplitude profile (c) of the acoustic wave at the surface of the granular layer for an excitation frequency of $1300 \mathrm{~Hz}$.

lowest amplitudes) on the reconstructed images (Fig. 9(a)). The second method consists in defining 10 different amplitude profiles (line trajectories from the source) of an image, pointing manually the first two minima of each profile and finally averaging the obtained distances between 2 successive minima over the 10 analyzed profiles. The last method consists in extracting automatically the distance between two successive minima of amplitude in the central trajectory line profile (as the one plotted in Fig. 9(a)). For this last method, a smoothing of the profiles is necessary for removing the high frequency noise and for focusing on the low frequency variations.

For an excitation frequency of $1100 \mathrm{~Hz}$, the half wavelength is equal to $33.4 \mathrm{~mm}$ (distance between two successive amplitude minima), which corresponds to a wavenumber of $94 \mathrm{rad} \mathrm{m}^{-1}$. For an excitation frequency of $1400 \mathrm{~Hz}$, the half wavelength is equal to $24.9 \mathrm{~mm}$, which corresponds to a wave number of $126 \mathrm{rad} \mathrm{m}^{-1}$. By measuring the spatial wavelengths for several excitation frequencies, the dispersion curve of the propagative mode at the surface is reconstructed in Fig. 14.

\section{COMPARISON WITH A FINITE ELEMENT MODELING OF THE PROBLEM}

Numerical simulations of the experiment were developed using finite element modeling. These simulations allow taking into consideration several important characteristics of the experiment, which cannot be accounted for all together using analytic developments: the specific source, the tridimensional nature of the problem, the finite depth of the layer, and the elasticity gradient. However, with a finite element modeling of the wave problem, it is possible to extract the data in the same manner as in experiments and reconstruct the dispersion curve accordingly, for meaningful comparison.

The simulated domain is a parallelepiped of $30 \mathrm{~cm}$ long, $20 \mathrm{~cm}$ wide, and $3.5 \mathrm{~cm}$ deep dimensions, including a homogeneous elastic medium, with the properties adapted to fit the known properties of the unconsolidated granular medium layer submitted to gravity. Using the previous studies on acoustics of granular media and the fairly well characterized elastic properties of granular layers, ${ }^{27,30,33,34,49,50}$ the depth-dependent Young modulus of the medium is chosen in the form

$$
E(z)=10^{8}\left(z^{1 / 4}+0.1 i|z+0.04|^{1 / 4}\right) .
$$

This Young modulus expression contains a real part and an imaginary part. The imaginary part (relatively smaller than the real part) corresponds to the acoustic energy dissipation, which is significant in weakly compressed granular media compared to usual elastic media. The real and imaginary parts of the modulus depend on the depth $z(z=0$ at the free surface of the granular medium). The dependence to the power $1 / 4$ is typical for granular media with low static pressures (usually lower than $100 \mathrm{kPa}$ ) and is in good agreement with sound speed measurements as a function of the static pressure. This power could also be chosen as $1 / 3$, in agreement with recent wave propagation experiments, ${ }^{27,30,33,34,49,50}$ but the sensitivity to this parameter was found to be not the most significant. Considering the imaginary part, there are no data available in the literature for such gravity loaded granular layers, except one qualitative tendency: the dissipation increases when the static pressure decreases (i.e., when closer to the free surface). The 0.1 factor is chosen so that the wave attenuation with the distance to the source is representative of the experimental results. The Poisson coefficient is taken equal to 0.3 , and the density of the medium is considered constant and equal to $1500 \mathrm{~kg} \mathrm{~m}^{-3}$.

The configuration and mesh of the layer are shown in Fig. 10. The acoustic source is modeled by a rigid and spherical bead of $3 \mathrm{~mm}$ diameter, located at a depth of $1 \mathrm{~cm}$, and oscillating with a $45^{\circ}$ angle relative to the normal to the surface, in the sagittal plane $(\mathrm{yOz})$, with a displacement amplitude of $1 \mathrm{~mm}$. The boundary conditions are rigid (zero displacement) at the bottom of the layer and free (zero stress) at the top, and absorbing conditions are used for the side boundaries. The mesh includes 45554 elements, and the simulation is made in the steady state frequency domain. A line at the surface is defined along the propagation direction $y$, with smaller elements, so that the surface displacement is extracted with a good spatial sampling.

In Figs. 11 and 12, two vibration modes of the medium are shown for two different excitation frequencies. The oscillations are rapidly attenuated as a function of the distance to the source. The spatial wavelengths depend indeed on the excitation frequency as experimentally observed and expected. In order to reconstruct the dispersion curve from these numerical data, the same method as for the experimental data was used. The displacement profiles in the $z$ direction along the measurement line (along the surface in the $y$ direction) are analyzed. For each excitation frequency, the distances between successive minima of the displacement amplitude are extracted and converted into wavelengths. Examples of these displacement profiles numerically obtained are plotted in Fig. 13. From these profiles, successive extrema 

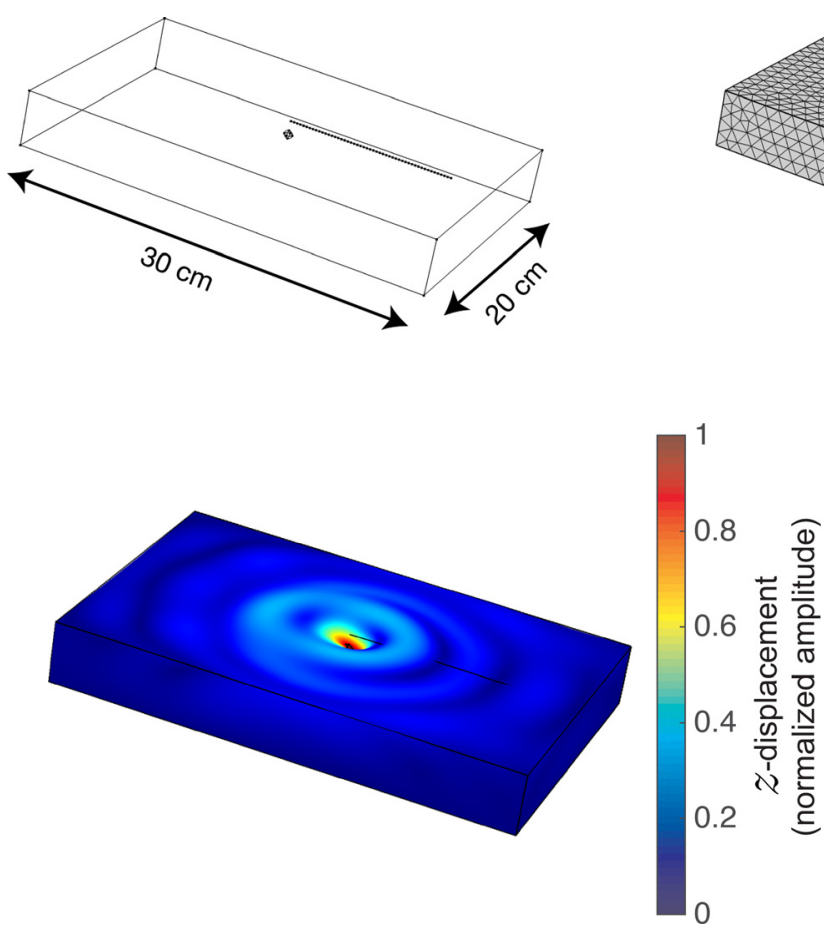

FIG. 11. Acoustic displacement amplitude obtained by FEM simulation of the wave problem for an excitation frequency of $1400 \mathrm{~Hz}$. The color scale corresponds to the z-direction displacement amplitude, while the surface deformation is proportional to the total displacement.

are automatically extracted and used to define a wavelength for the field, e.g., the distance between the first minimum and the following maximum is the quarter of a wavelength. The values of the extracted wavelengths for several couples of extrema, at each frequency, lead to the dispersion data in Fig. 14.

In Figure 14, the points of the dispersion curve from experimental results and the ones from numerical results are plotted together. A relatively good agreement is observed for the estimation of the dispersion curve. Note that the processing method for the FEM results makes use of the distances between several minima and maxima, while in experiments, data were extracted only from the two first minima. All the data extracted from FEM are provided because they are available, but in principle, only the average of the FEM data

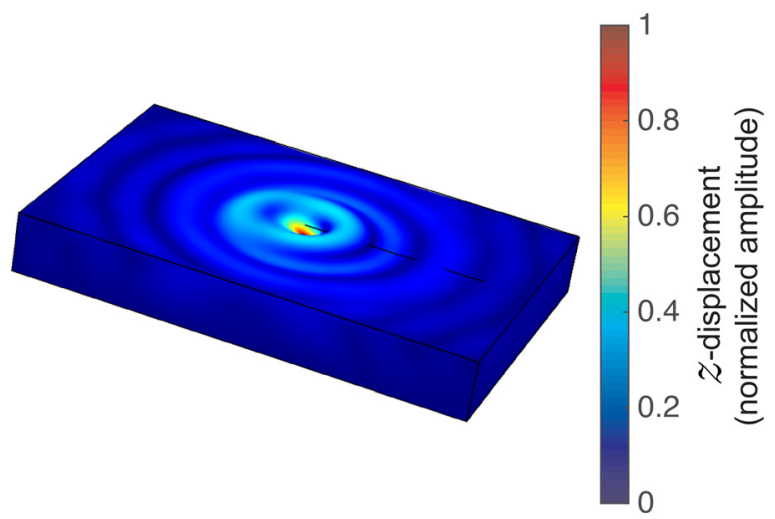

FIG. 12. Acoustic displacement amplitude obtained by FEM simulation of the wave problem for an excitation frequency of $1700 \mathrm{~Hz}$. The color scale corresponds to the $\mathrm{z}$-direction displacement amplitude, while the surface deformation is proportional to the total displacement.
FIG. 10. Geometrical configuration and mesh for the FEM simulation. "extrema 1-2" and "extrema 2-3" should be compared to the experimental data. The experimental points at $1500 \mathrm{~Hz}$ are clearly shifted in regard to the experimental tendency and in regard of the numerical simulation results. We could not find a clear explanation for this observation, except the difficulty to define the experimental wavelength when signals are too much noisy or do not exhibit a sufficient number of extrema in the analyzed zone (as observed with the numerical results for frequencies below $1100 \mathrm{~Hz}$ ). Results in Fig. 14 confirm the feasibility of qualitative and even quantitative measurements by three color digital holography, for the propagation of dispersive modes in unconsolidated granular layers. This
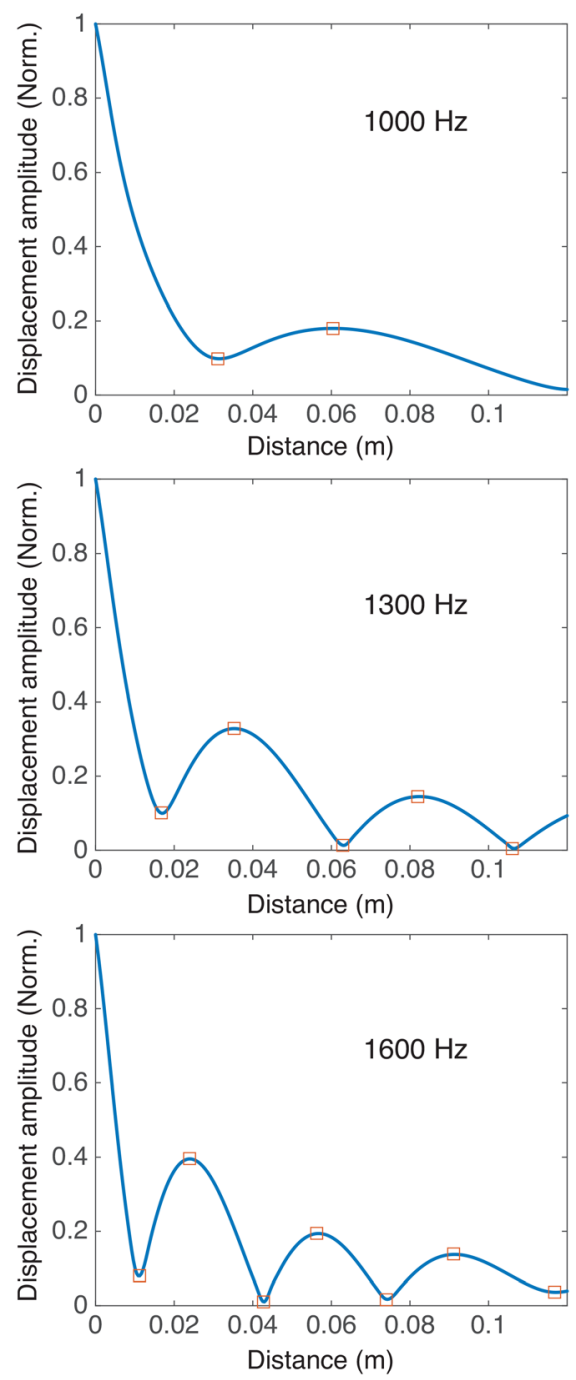

FIG. 13. Acoustic displacement amplitude profiles obtained by FEM simulation for excitation frequencies of 1000, 1300, and $1600 \mathrm{~Hz}$. Displacement amplitudes are normalized to their value at the source location. 


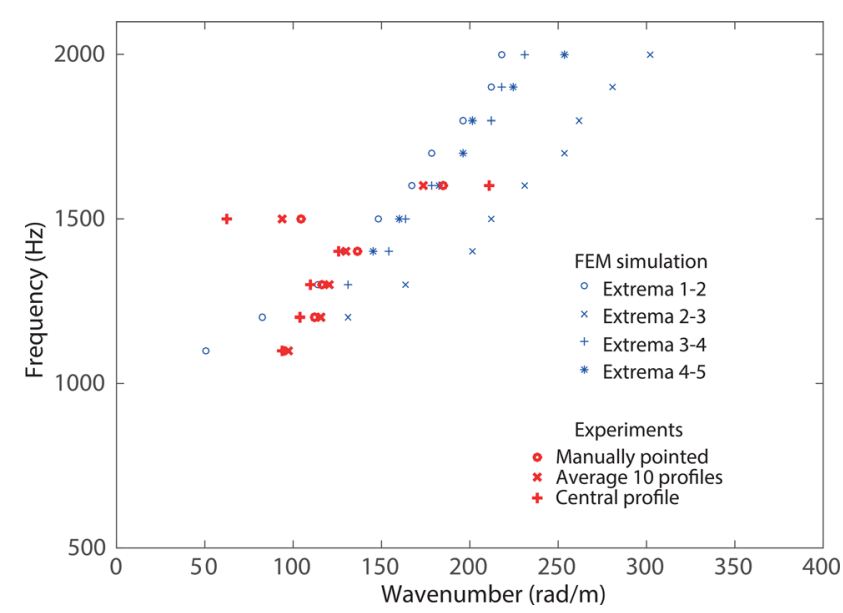

FIG. 14. Superposition of the dispersion curves for the response of the medium to excitation frequencies from $1100 \mathrm{~Hz}$ to $2000 \mathrm{~Hz}$, obtained by FEM simulation (blue) and experimentally (red).

gives opportunity for studying problems with complex geometries, where point-to-point measurements are difficult to implement.

\section{IN-PLANE ACOUSTIC DISPLACEMENTS}

By using the experimental method and Equation (10), acoustic amplitudes along the $x$ and $y$ directions can also be measured. Figure 15 shows acoustic displacement amplitudes measured for an excitation frequency of $1200 \mathrm{~Hz}$. Displacements are observed in the two directions, certainly because the wave field emitted from the source is cylindrically divergent. Although the excited modes are dominantly corresponding to sagittal modes and longitudinal modes, this cylindrical field structure leads to comparable acoustic displacement amplitudes along the $x$ and $y$ directions. Acoustic displacement amplitudes are around 100 and 50 nanometers, respectively.

\section{INTERACTION WITH A BURIED OBJECT}

The holographic method with its full-field capability is useful to visualize the influence of objects buried inside the medium on the elastic wave field. As an example, Figure 16 shows the acoustic displacement amplitude measured for an excitation frequency of $1500 \mathrm{~Hz}$, with and without the inclusion of a $5 \mathrm{~mm}$ in diameter steal cylinder buried inside the layer. The top of the buried cylinder is located just a few $\mathrm{mm}$ below the granular surface so that it is not visible.
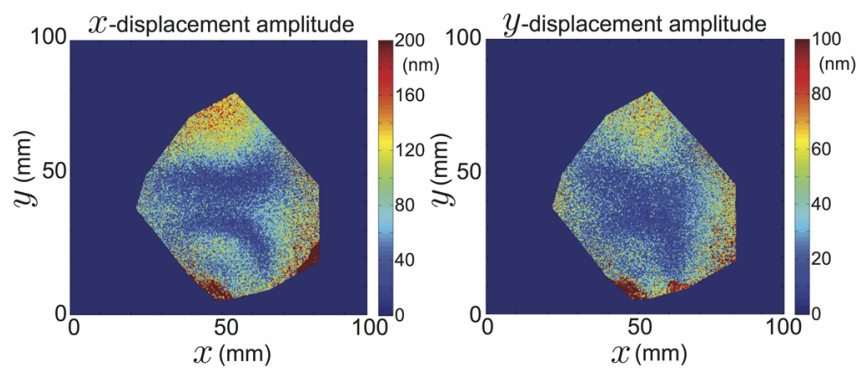

FIG. 15. Amplitudes of the acoustic displacement along the $x$ (left) and $y$ (right) directions for an excitation frequency of $1200 \mathrm{~Hz}$.

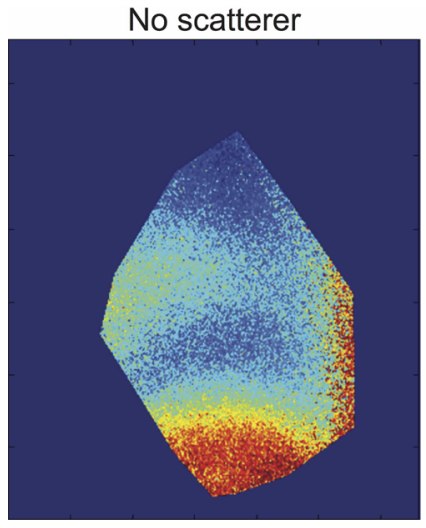

With a buried scatterer

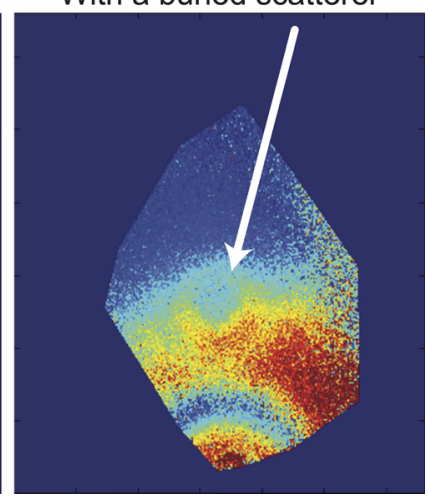

FIG. 16. Acoustic displacement amplitude along the $z$ direction (out-ofplane), without (left) and with (right) a buried scatterer (a steal cylinder), for an excitation frequency of $1500 \mathrm{~Hz}$.

The obstacle position is clearly seen, as well as the acoustic wave diffracted by the object.

This qualitative result shows the capabilities of the method for the detection of buried scatterers. The experimental analysis of wave propagation in periodic buried structures, and of wave scattering by simple shape scatterers in waveguides with in-depth elasticity gradients, could be future applications of the method.

\section{CONCLUSION}

We have reported on the use of digital three-color holography to investigate acoustic waves propagating along the surface of a granular medium layer. The presented technique leads to a simultaneous recording and measurement of the $3 \mathrm{D}$ acoustic displacement associated with the propagating modes and provided full-field measurements. In addition, not only the amplitude but also the phase of the acoustic wave can be visualized.

The propagation of acoustic waves along the surface of a granular layer is dispersive. The holographic reconstruction could provide useful information on the acoustic wave displacement in the $z$ direction (out-of-plane), for frequencies between $800 \mathrm{~Hz}$ and $2000 \mathrm{~Hz}$ in the studied layer. Amplitudes of the acoustic wave displacement at the surface are found to be of few hundredths of nanometers. The quantitative information of the holographic reconstruction is shown to be useful for providing the dispersion curve of the propagating modes. The experimentally obtained dispersion curve is compared successfully to the one obtained with a finite element simulation of an equivalent elastic wave problem. The 3D character of the wave displacement field is exhibited, and transverse (in-plane) components are visualized. Finally, the inclusion of a buried obstacle was detected by the 3D holographic probe, opening the perspective of a full-field study of the interaction between elastic wave modes and buried objects in granular layers.

\section{ACKNOWLEDGMENTS}

This research was funded by the French National Agency for Research (ANR) under Grant Agreement No. ANR 2010 BLAN 0302. 
${ }^{1}$ E. Cuche, F. Bevilacqua, and C. Depeursinge, "Digital holography for quantitative phase-contrast imaging,” Opt. Lett. 24(5), 291-293 (1999).

${ }^{2}$ C. Mann, L. Yu, C-M. Lo, and M. Kim, "High-resolution quantitative phase-contrast microscopy by digital holography," Opt. Express 13(22), 8693-8698 (2005).

${ }^{3}$ B. Bhaduri, H. Pham, M. Mir, and G. Popescu, "Diffraction phase microscopy with white light," Opt. Lett. 37(6), 1094-1096 (2012).

${ }^{4}$ P. Girshovitz and N. T. Shaked, "Compact and portable low-coherence interferometer with off-axis geometry for quantitative phase microscopy and nanoscopy," Opt. Express 21(5), 5701-5714 (2013).

${ }^{5}$ G. Rajshekhar, B. Bhaduri, C. Edwards, R. Zhou, L. L. Goddard, and G. Popescu, "Nanoscale topography and spatial light modulator characterization using wide-field quantitative phase imaging," Opt. Express 22(3), 3432-3438 (2014).

${ }^{6}$ P. Ferraro, D. Alferi, S. De Nicola, L. De Petrocellis, A. Finizio, and G. Pierattini, "Quantitative phase-contrast microscopy by a lateral shear approach to digital holographic image reconstruction," Opt. Lett. 31(10), 1405-1407 (2006).

${ }^{7}$ E. Shaffer, C. Moratal, P. Magistretti, P. Marquet, and C. Depeursinge, "Label-free second-harmonic phase imaging of biological specimen by digital holographic microscopy," Opt. Lett. 35(24), 4102-4104 (2010).

${ }^{8}$ H. Gabaj and N. T. Shaked, "Dual-channel low-coherence interferometry and its application to quantitative phase imaging of fingerprints," Opt. Express 20(24), 26906-26912 (2012).

${ }^{9}$ A. B. Parthasarathy, K. K. Chu, T. N. Ford, and J. Mertz, "Quantitative phase imaging using a partitioned detection aperture," Opt. Lett. 37(19), 4062-4064 (2012).

${ }^{10}$ C. J. Mann, P. R. Bingham, V. C. Paquit, and K. W. Tobin, "Quantitative phase imaging by three-wavelength digital holography," Opt. Express 16(13), 9753-9764 (2008).

${ }^{11}$ P. Picart, M. Malek, V. C. Paquit, and K. W. Tobin, "Complex field recovering from in-line digital holography," Opt. Lett. 38(17), 3230-3232 (2013).

${ }^{12}$ J.-M. Desse, P. Picart, and P. Tankam, "Digital three-color holographic interferometry for flow analysis," Opt. Express 16(8), 5471-5480 (2008).

${ }^{13}$ T. Kakue, R. Yonesaka, T. Tahara, Y. Awatsuji, N. Kenzo, S. Ura, T. Kubota, and O. Matoba, "High-speed phase imaging by parallel phaseshifting digital holography," Opt. Lett. 36(21), 4131-4133 (2011).

${ }^{14}$ J.-M. Desse, P. Picart, and P. Tankam, "Digital color holography applied to fluid and structural mechanics," Opt. Lasers Eng. 50(1), 18-28 (2012).

${ }^{15} \mathrm{~S}$. Seebacher, W. Osten, T. Baumbach, and W. O. Jüptner, "The determination of material parameters of microcomponents using digital holography," Opt. Lasers Eng. 36(2):103-126 (2001).

${ }^{16}$ S. Grilli, M. Paturzo, L. Miccio, and P. Ferraro, "In situ investigation of periodic poling in congruent $\mathrm{LiNbO3}$ by quantitative interference microscopy," Meas. Sci. Technol. 19(7), 074008 (2008).

${ }^{17}$ I. Yamaguchi, T. Ida, M. Yokota, and K. Yamashita, "Surface shape measurement by phase shifting digital holography with a wavelength shift," Applied Optics 45(29), 7610-7616 (2006).

${ }^{18}$ T. Nomura, B. Javidi, S. Murata, E. Nitanai, and T. Numata, "Polarization imaging of a $3 \mathrm{~d}$ object by use of on-axis phase-shifting digital holography," Opt. Lett. 32(5), 481-483 (2007).

${ }^{19}$ Y. Morimoto, T. Nomura, M. Fujigaki, S. Yoneyama, and I. Takahashi, "Deformation measurement by phase shifting digital holography," Exp. Mech. 45(1), 65-70 (2005)

${ }^{20}$ P. Tankam, Q. Song, M. Karray, J. C. Li, J-M. Desse, and P. Picart, "Realtime three-sensitivity measurements based on three-color digital fresnel holographic interferometry," Opt. Lett. 35(12), 2055-2057 (2010).

${ }^{21}$ Y. Fu, G. Pedrini, and W. Osten, "Vibration measurement by temporal fourier analysis of a digital hologram sequence," Appl. Opt. 46(23), 5719-5727 (2007).

${ }^{22}$ M. Leclercq, M. Karray, V. Isnard, F. Gautier, and P. Picart, "Evaluation of surface acoustic waves on the human skin using quasi-time-averaged digital fresnel holograms," Appl. Opt. 52(1), A136-A146 (2013).

${ }^{23}$ F. Joud, F. Verpillat, F. Laloé, M. Atlan, J. Hare, and M. Gross, "Fringefree holographic measurements of large-amplitude vibrations," Opt. Lett. 34(23), 3698-3700 (2009).

${ }^{24}$ B. Samson, F. Verpillat, M. Gross, and M. Atlan, "Video-rate laser doppler vibrometry by heterodyne holography," Opt. Lett. 36(8), 1449-1451 (2011).

${ }^{25}$ P. Tankam, P. Picart, D. Mounier, J.-P. Boileau, V. Tournat, and V. E. Gusev, "Investigation of dynamic of unconsolidated materials using twocolor digital holography," EPJ Web Conf. 6, 30004 (2010).
${ }^{26}$ B. Andreotti, Y. Forterre, and O. Pouliquen, Granular Media: Between Fluid and Solid (Cambridge University Press, 2013).

${ }^{27}$ X. Jacob, V. Aleshin, V. Tournat, P. Leclaire, W. Lauriks, and V. E. Gusev, "Acoustic probing of the jamming transition in an unconsolidated granular medium," Phys. Rev. Lett. 100(15), 158003 (2008).

${ }^{28}$ V. E. Gusev, V. Aleshin, and V. Tournat, "Acoustic waves in an elastic channel near the free surface of granular media," Phys. Rev. Lett. 96, 214301 (2006).

${ }^{29} \mathrm{~V}$. Aleshin, V. Gusev, and V. Tournat, "Acoustic modes propagating along the free surface of granular media," J. Acoust. Soc. Am. 121(5), 2600-2611 (2007).

${ }^{30}$ L. Bodet, X. Jacob, V. Tournat, and R. Mourgues, "Elasticity profile of an unconsolidated granular medium inferred from guided waves: Toward acoustic monitoring of analogue models," Tectonophysics 496(1-4), 99-104 (2010).

${ }^{31}$ L. Bonneau, B. Andreotti, and E. Clément, "Surface elastic waves in granular media under gravity and their relation to booming avalanches," Phys. Rev. E 75(1 Pt 2), 016602 (2007).

${ }^{32}$ L. Bonneau, B. Andreotti, and E. Clément, "Evidence of rayleigh-hertz surface waves and shear stiffness anomaly in granular media," Phys. Rev. Lett. 101(11), 118001 (2008).

${ }^{33}$ L. Bodet, A. Dhemaied, R. Martin, R. Mourgues, F. Rejiba, and V. Tournat, "Small-scale physical modeling of seismic-wave propagation using unconsolidated granular media," Geophysics 79(6), T323-T339 (2014).

${ }^{34}$ P. Bergamo, L. Bodet, L. V. Socco, R. Mourgues, and V. Tournat, "Physical modelling of a surface-wave survey over a laterally varying granular medium with property contrasts and velocity gradients," Geophys. J. Int. 197(1), 233-247 (2014).

${ }^{35} \mathrm{~J}$. W. Goodman, Introduction to Fourier Optics, 2nd ed. (McGraw-Hill Education, 1996).

${ }^{36} \mathrm{U}$. Schnars and W. Jüptner, "Direct recording of holograms by a ccd target and numerical reconstruction,” Appl. Opt. 33(2), 179-181 (1994).

${ }^{37} \mathrm{P}$. Picart and J. Leval, "General theoretical formulation of image formation in digital fresnel holography,” J. Opt. Soc. Am. 25(7), 1744-1761 (2008).

${ }^{38}$ T. Kreis, M. Adams, and W. Jüptner, "Methods of digital holography: A comparison," SPIE Proc. 3098, 224-233 (1997).

${ }^{39} \mathrm{~K}$. A. Stetson, "Fringe interpretation for hologram interferometry of rigidbody motions and homogeneous deformations," JOSA 64(1), 1-10 (1974).

${ }^{40}$ J. C. Li, P. Tankam, Z. J. Peng, and P. Picart, "Digital holographic reconstruction of large objects using a convolution approach and adjustable magnification," Opt. Lett. 34(5), 572-574 (2009).

${ }^{41}$ U. Schnars, T. M. Kreis, and W. O. Jüptner, "Digital recording and numerical reconstruction of holograms: Reduction of the spatial frequency spectrum," Opt. Eng. 35(4), 977-982 (1996).

${ }^{42}$ J. Mundt and T. Kreis, "Digital holographic recording and reconstruction of large scale objects for metrology and display," Opt. Eng. 49(12), 125801 (2010).

${ }^{43}$ S. De Nicola, A. Finizio, and G. Pierattini, "Recovering correct phase information in multi wavelength digital holographic microscopy by compensation for chromatic aberrations," Opt. Lett. 30(20), 2706-2708 (2005).

${ }^{44}$ P. Ferraro, S. Grilli, L. Miccio, D. Alfieri, S. De Nicola, A. Finizio, and B. Javidi, "Full color 3-D imaging by digital holography and removal of chromatic aberrations," J. Display Technol. 4(1), 97-100 (2008).

${ }^{45}$ M. Leclercq and P. Picart, "Method for chromatic error compensation in digital color holographic imaging," Opt. Express 21(22), 26456-26467 (2013).

${ }^{46}$ P. Ferraro, S. De Nicola, G. Coppola, A. Finizio, D. Alfieri, and G. Pierattini, "Controlling image size as a function of distance and wavelength in fresnel-transform reconstruction of digital holograms," Opt. Lett. 29(8), 854-856 (2004).

${ }^{47} \mathrm{P}$. Tankam and P. Picart, "Use of digital color holography for crack investigation in electronic components," Opt. Lasers Eng. 49(11), 1335-1342 (2011).

${ }^{48}$ J. Leval, P. Picart, J.-P. Boileau, and J.-P. Dalmont, "Full field vibrometry with digital fresnel holography," Appl. Opt. 44(27), 5763-5772 (2005).

${ }^{49}$ J.-B. Legland, V. Tournat, O. Dazel, A. Novak, and V. E. Gusev, "Linear and nonlinear Biot waves in a noncohesive granular medium slab: Transfer function, self-action, second harmonic generation," J. Acoust. Soc. Am. 131(6), 4292-4303 (2012).

${ }^{50} \mathrm{~V}$. Tournat and V. E. Gusev, "Acoustics of unconsolidated granular media: An overview of recent results and several open problems," Acta-Acust. Acust. 96(2), 208-224 (2010). 